PROCEEDINGS OF THE

AMERICAN MATHEMATICAL SOCIETY

Volume 128, Number 3, Pages 803-812

S 0002-9939(99)05042-X

Article electronically published on July 6, 1999

\title{
TRANSLATION INVARIANT JULIA SETS
}

\author{
DAVID BOYD
}

(Communicated by Albert Baernstein II)

\begin{abstract}
We show that if the Julia set $J(f)$ of a rational function $f$ is invariant under translation by one and infinity is a periodic or preperiodic point for $f$, then $J(f)$ must either be a line or the Riemann sphere.
\end{abstract}

\section{INTRODUCTION}

One of the more attractive aspects of Julia sets for rational functions is the display of self-similarity on many different scales. On a global scale, Beardon classified in [2] the finite order rotational symmetries of Julia sets of polynomials. An earlier result along these lines was presented by Baker and Eremenko in [1]. The question arises of what kind of global symmetries the Julia set of a general rational function may possess, i.e., if we assume $\phi$ is a Möbius, or linear fractional, transformation such that $\phi(J(f))=J(f)$, and hence $\phi(F(f))=F(f)$ where $J(f)$ is the Julia set of $f$ and $F(f)$ is the Fatou set of $f$, what can we conclude about $f$ ? There are examples of rational functions $f$ with $J(f)=\overline{\mathbb{C}}$ (see [3], §4.3) which is invariant under all Möbius transformations. The function $f(z)=z-z^{-1}$ has $J(f)=\mathbb{R} \cup\{\infty\}$ which is for example invariant under all Möbius transformations with real coefficients, and in particular under translation by all real numbers. Recall the following conjecture.

Conjecture 1. Let $f$ be a rational function of degree at least two and let $\phi$ be a Möbius transformation of infinite order, i.e., $\phi^{n} \neq \phi$ for all $n \geq 2$, such that $\phi(J(f))=J(f)$. Then $J(f)$ is Möbius equivalent to $\overline{\mathbb{C}}$, a circle, or a closed line segment.

We discuss the extent to which this conjecture has been treated in the literature in Section 2. In this paper we give a partial proof of Conjecture 1.

Theorem 1. Let $f$ be a rational function of degree at least two such that $J(f)+1=$ $J(f)$ and such that infinity is either periodic or preperiodic, i.e., there exist a point $w_{0} \in \overline{\mathbb{C}}$ and integers $n, m \geq 1$ such that $f^{n}(\infty)=w_{0}$ and $f^{m}\left(w_{0}\right)=w_{0}$. Then $J(f)$ is either all of $\overline{\mathbb{C}}$ or is a horizontal line.

The author would like to thank Noel Baker for his valuable suggestions which led to a strengthening of Theorem 1 in the case when infinity is a strictly preperiodic point and a critical point.

Received by the editors November 10, 1997 and, in revised form, April 27, 1998.

1991 Mathematics Subject Classification. Primary 30D05.

Research supported by a Department of Education GAANN fellowship and by the Research Board of the University of Illinois at Urbana-Champaign.

(C)1999 American Mathematical Society 
The results of this paper are part of the author's Ph.D. thesis, done under the supervision of Aimo Hinkkanen.

\section{TRANSLATION INVARIANT JULIA SETS}

We first prove the following lemma.

Lemma 1. Let $f$ be a rational function of degree at least two such that $J(f)$ is Möbius equivalent to a circle in $\mathbb{C}$. Then $J(f)$ cannot contain a critical point of $f$.

Proof. Assume that this is not the case. By conjugating $f$ by the appropriate Möbius transformation, we may assume that $J(f)=\mathbb{R} \cup\{\infty\}$ and that $f$ has the expansion

$$
f(z)=1+a z^{k}+O\left(z^{k+1}\right), k \geq 2, a \neq 0,
$$

in a neighborhood of 0 . Then the image under $f$ of a set of the form

$$
D=\left\{z=r e^{i \theta}: 0<r<\delta, 0<\theta<\pi\right\}
$$

for small $\delta$ contains a set of the form

$$
D^{\prime}=\left\{z=1+r e^{i \theta}: 0<r<\delta^{\prime}<|a| \delta^{k}, \arg a<\theta<\arg a+3 \pi / 2\right\} .
$$

In particular, we would have points of $F(f)$ mapping onto points of $J(f)$, which is impossible.

Let $\phi$ be a parabolic Möbius transformation, i.e., $\phi$ has a single fixed point in $\overline{\mathbb{C}}$. Then there is another Möbius transformation $\psi$ such that $\left(\psi \circ \phi \circ \psi^{-1}\right)(z)=z+1$. Let $g$ be a rational function of degree at least two such that $\phi(J(g))=J(g)$. If $f=\psi \circ g \circ \psi^{-1}$, then by [3], Theorem 3.1.4,

$$
J(f)+1=\left(\psi \circ \phi \circ \psi^{-1}\right)(J(f))=(\psi \circ \phi)(J(g))=\psi(J(g))=J(f) .
$$

Using this observation, the following material has an analogue for rational functions $f$ such that $\phi(J(f))=J(f)$ for a parabolic Möbius transformation $\phi$.

Remark 1. As $J(f)$ is closed in $\overline{\mathbb{C}}$, the assumption $J(f)+1=J(f)$ implies that $\infty \in J(f)$.

Remark 2. By passing to an iterate of $f$, we may assume that if $F(f) \neq \emptyset$, then every component of $F(f)$ is either fixed by $f$ or iterates onto a component of $F(f)$ fixed by $f$ by Sullivan's no-wandering-domains theorem.

Remark 3. If $U$ is a component of $F(f)$, then so is the set $U+1=\{z+1: z \in U\}$, for by the assumed invariance, $U+1$ is a connected subset of $F(f)$, hence is contained in some component $V$ of $F(f)$. However, the boundary of $U+1$ is a subset of $J(f)$ and so $U+1$ must coincide with $V$. Similarly, $U+n$ is a component of $F(f)$ for any integer $n$. Note that by the above observation, if $U+n$ meets $U$, they must coincide. Further, we have the following lemma.

Lemma 2. Let $U$ be a component of the Fatou set of a rational function $f$ such that $J(f)+1=J(f)$. If $U+n=U$ for any non-zero integer $n$, then $U+1=U$ and hence $U+n=U$ for all integers $n$. 
Proof. Take any point $z_{0} \in U$. By assumption, $z_{0}+n$ also lies in $U$. Thus we may connect $z_{0}$ and $z_{0}+n$ by a simple, analytic curve $\gamma$ lying completely in $U$. Construct the set $\Gamma=\bigcup_{m=-\infty}^{\infty}(\gamma+m n)$, which is a connected subset of $U$. As $\gamma$ is bounded, it contains points $z_{1}$ and $z_{2}$ on which the function $\operatorname{Im}(z)$ achieves a minimum and maximum, respectively. Hence $\Gamma$ is contained in the strip $S=\{z$ : $\left.\operatorname{Im}\left(z_{1}\right) \leq \operatorname{Im}(z) \leq \operatorname{Im}\left(z_{2}\right)\right\}$. The set $\Gamma+1$ is a connected subset of $S$, unbounded in both the positive and negative real directions. Hence $(\Gamma+1) \cap \gamma \neq \emptyset$. In particular, this shows that $U \cap(U+1) \neq \emptyset$ and so $U+1=U$.

Conjecture 1 would follow from [5], Theorem 2. p. 1127, where it was claimed that the number of rational functions of the same degree sharing the same nonsimple Julia set is finite. This theorem is also referred to in [4], p. 2181. However the proof of this theorem contains an acknowledged gap.

2.1. Proof of Theorem 1. Before we begin the proof of Theorem 1, we make some additional observations implied by the assumptions of the theorem.

Remark 4. Since $J(f)=J\left(f^{n}\right)$ for all $n \in \mathbb{N}$ by [3], Theorem 3.1.5, by replacing $f$ with a suitable iterate, we may assume that $f(\infty)=w_{0}$ and $f\left(w_{0}\right)=w_{0}$. Thus we are assuming infinity is either fixed or the image of infinity is fixed by $f$. Now since $\infty \in J(f)$, then $w_{0} \in J(f)$ as well. Thus $w_{0}$ cannot be a superattracting or attracting fixed point of $f$.

If $w_{0}=\infty$, then $f$ has the expansion $f(z)=z / \lambda+\sum_{k=0}^{\infty} b_{k} / z^{k}$ in a neighborhood of infinity with $|\lambda| \geq 1$ since the fixed point is neither superattracting nor attracting. When $\lambda \neq 1$, by replacing $f$ with its conjugate by the map $z \mapsto z+a$ where $a=\lambda b_{0} /(1-\lambda)$, which preserves the property that $J(f)+1=J(f)$, we may assume that $b_{0}=0$. Hence when $\lambda \neq 1$, we may assume we have the estimates

$$
\begin{aligned}
f(z) & =z / \lambda+O(1 / z), \\
f^{-1}(z) & =\lambda z+O(1 / z)
\end{aligned}
$$

in a neighborhood of $\infty$.

If $w_{0} \in \mathbb{C}$ and infinity is not a critical point for $f$, we have the estimates

$$
f(z)=f_{\infty}(z)=w_{0}+a / z+O\left(1 / z^{2}\right)
$$

in a neighborhood of infinity, and

$$
f(z)=f_{w_{0}}(z)=\lambda\left(z-w_{0}\right)+w_{0}+O\left(\left(z-w_{0}\right)^{2}\right)
$$

in a neighborhood of $w_{0}$. Again, since $w_{0}$ cannot be superattracting or attracting, $|\lambda| \geq 1$. If $\lambda=1$, define $g$ to be

$$
g(z)=\left(f_{\infty}^{-1} \circ f_{w_{0}} \circ f_{\infty}\right)(z)=z+O(1)
$$

in a neighborhood of infinity. If $\lambda \neq 1$, we may choose a translation $\varphi$ as above so that the function

$$
\begin{aligned}
g(z) & =\left(\varphi \circ f_{\infty}^{-1} \circ \varphi^{-1}\right) \circ\left(\varphi \circ f_{w} \circ \varphi^{-1}\right) \circ\left(\varphi \circ f_{\infty} \circ \varphi^{-1}\right)(z) \\
& =\left(\varphi \circ f_{\infty}^{-1} \circ f_{w_{0}} \circ f_{\infty} \circ \varphi^{-1}\right)(z)
\end{aligned}
$$

is an analytic function and has the expansion $g(z)=z / \lambda+O(1 / z)$ in a neighborhood of infinity. If we replace $f$ by $\varphi \circ f \circ \varphi^{-1}, g$ will preserve $F(f)$ and $J(f)$ where defined. Note that where defined, $g^{n}=f_{\infty}^{-1} \circ f_{w_{0}}^{n} \circ f_{\infty}$. 
If $w_{0} \in \mathbb{C}$ and infinity is a critical point for $f$, then we have the estimates

$$
f(z)=f_{\infty}(z)=w_{0}+a / z^{k}+O\left(1 / z^{k+1}\right)
$$

in a neighborhood of infinity where $a \neq 0$ and $k \geq 2$ and

$$
f(z)=f_{w_{0}}(z)=\lambda\left(z-w_{0}\right)+w_{0}+O\left(\left(z-w_{0}\right)^{2}\right)
$$

in a neighborhood of $w_{0}$ where, again, $|\lambda| \geq 1$. Given a choice of $\lambda^{1 / k}$ there is a function $g$ analytic in a neighborhood of infinity such that

$$
g(z)=z / \lambda^{1 / k}+O(1)
$$

and $f_{\infty} \circ g=f_{w_{0}} \circ f_{\infty}$. We see this as follows. We may write

$$
f_{\infty}(z)=w_{0}+\frac{1}{z^{k}}(a+O(1 / z))=w_{0}+(F(z))^{k}
$$

where $F(z)=a^{1 / k} / z+O\left(1 / z^{2}\right)$ in a neighborhood of infinity and we may similarly write

$$
\left(f_{w_{0}} \circ f_{\infty}\right)(z)=w_{0}+\frac{1}{z^{k}}(\lambda a+O(1 / z))=w_{0}+(G(z))^{k}
$$

where $G(z)=(\lambda a)^{1 / k} / z+O\left(1 / z^{2}\right)$ in a neighborhood of infinity. Let $g(z)=$ $\left(F^{-1} \circ G\right)(z)=z / \lambda^{1 / k}+O(1)$ in a suitable neighborhood of infinity. Note that $f_{\infty} \circ g=f_{w_{0}} \circ f_{\infty}$ and hence $g$ preserves $F(f)$ and $J(f)$ where defined. When our choice of $\lambda^{1 / k} \neq 1$, we may replace $f$ and $g$ by a suitable conjugate by a translation as above so that

$$
g(z)=z / \lambda^{1 / k}+O(1 / z)
$$

in a neighborhood of infinity and $g$ still preserves $F(f)$ and $J(f)$ where defined. Also, $f_{\infty} \circ g^{n}=f_{w_{0}}^{n} \circ f_{\infty}$ where defined.

We continue the proof of Theorem 1 using the assumptions in the above remarks. As $w_{0} \in J(f)$ is fixed, we break up the proof into separate lemmas depending on whether $w_{0}$ is a repelling, parabolic, or Cremer point. We first prove a lemma to treat the repelling case.

Lemma 3. Let $f$ be a rational function of degree at least two such that $J(f)+1=$ $J(f)$ and let $g$ be an analytic function defined in a neighborhood of infinity so that

$$
\begin{aligned}
g(z) & =z / \lambda+O(1 / z), \\
g^{-1}(z) & =\lambda z+O(1 / z)
\end{aligned}
$$

where $|\lambda|>1$ and where defined, $g$ leaves both $F(f)$ and $J(f)$ invariant. If $F(f) \neq$ $\emptyset$, then $\lambda$ is real.

We will apply this lemma as follows. When infinity is a repelling fixed point, set $g=f$. When $w_{0}=f(\infty) \in \mathbb{C}$ is a repelling fixed point and infinity is not a critical point, define $g$ by (2.7). When $w_{0}=f(\infty) \in \mathbb{C}$ is a repelling fixed point and infinity is a critical point, define $g$ by (2.10) where we identify $\lambda$ with $\lambda^{1 / k}$.

Proof. We use the map $g$ to show that $F(f)$ contains disks of arbitrarily large radius. Assume that this is not the case, i.e., there exist a positive number $r$ and a point $z_{0}$ such that the disk $\Delta=\Delta\left(z_{0}, r\right) \subset F(f)$, yet given any $w \in \mathbb{C}$ and $\delta>0$, then $\Delta(w, r+\delta) \cap J(f) \neq \emptyset$. Let $L=\{z:|z|>M\}$ be a neighborhood of infinity such that $g^{-1}(L) \subset L$ and where the estimate $\left|g^{-1}(z)-\lambda z\right|<(|\lambda|-1) r / 4$ holds. By the invariance under translation by one, we may choose the integer $n$ 
large enough so that $\Delta+n \subset L \cap F(f)$. With this estimate, $g^{-1}(\Delta+n)$ contains a disk of radius $(|\lambda|+1) r / 2>r$. However, by the invariance of $F(f)$ under $g$, we have that $g^{-1}(\Delta+n) \subset F(f)$. This is a contradiction. Hence $F(f)$ contains disks of arbitrarily large radius.

Assume that $\theta=\arg 1 / \lambda$ is neither 0 nor $\pi$. We may assume that $0<\theta<\pi$. As $F(f)$ contains disks of arbitrarily large radius, we may choose a disk $\Delta\left(z_{0}, r\right) \subset F(f)$ such that the union $\bigcup_{n=-\infty}^{\infty} \Delta\left(z_{0}+n, r\right)$ contains the strip $S=\{z: \mid \operatorname{Im}(z)-$ $\left.\operatorname{Im}\left(z_{0}\right) \mid<4\right\}$. Note that $S \subset F(f)$. As $0<\theta<\pi$, we may replace $\Delta\left(z_{0}, r\right)$ by a disk contained in $g^{-1}\left(\Delta\left(z_{0}+k, r\right)\right)$ so that the corresponding strip $S$ is contained in a neighborhood of infinity where $\left|g^{-1}(z)-\lambda z\right|<1 / 10$. Then $g^{-1}(S)$, and hence $F(f)$, contain a strip $T$ of the form $T=\{z: \operatorname{dist}(z, \mathcal{L})<3\}$ where $\mathcal{L}$ is the line $\left\{\lambda z_{0}+t \lambda: t \in \mathbb{R}\right\}$. For such a strip $T, \mathbb{C}=\bigcup_{n=-\infty}^{\infty}(T+n) \subset F(f)$, since for any point $w \in \mathbb{C}$ there is an integer $n$ so that $\operatorname{dist}(w, \mathcal{L}+n)<1$ and so $w \in T+n$. This is impossible, for the Julia set for any rational function of degree at least two must contain infinitely many points. Hence $\lambda$ is real. We may assume that $\lambda>1$.

Lemma 4. Let $f$ and $g$ be as in Lemma 3. Then $J(f)$ is a horizontal line if $F(f) \neq \emptyset$.

Proof. Recall that the conclusion that $J(f)$ is a horizontal line is incompatible with the assumption that there is a critical point in $J(f)$ by Lemma 1.

We are still assuming the estimates (2.11) and (2.12), where we may take $\lambda>1$ by Lemma 3. With these, if we assume $F(f) \neq \emptyset$, we will show that $J(f)=\mathbb{R} \cup\{\infty\}$.

First we show that for any point $z_{0} \in J(f)$, the horizontal line through $z_{0}$ is contained in $J(f)$. Assume the contrary, i.e., there exist a point $z_{0} \in J(f)$ and a point $w \in F(f) \cap\left[z_{0}, z_{0}+1\right]$ where $\left[z_{0}, z_{0}+1\right]$ is the closed line segment between $z_{0}$ and $z_{0}+1$. As $F(f)$ is open, there is a disk $\Delta(w, r) \subset F(f)$. We may choose positive integers $n$ and $m$ large enough so that $g^{-m}(\Delta(w+n, r))$ contains the disk $\Delta\left(g^{-m}(w+n), 2\right)$. We may further assume that the estimate $\left|g^{-m}(z)-\lambda^{m} z\right|<1 / 10$ holds on $z_{0}+n$ and $w+n$. As $\lambda$ is real, there exists an integer $k$ so that

$$
g^{-m}\left(z_{0}+n\right) \in \Delta\left(g^{-m}(w+n), 2\right)-k,
$$

since

$$
\left|\operatorname{Im}\left(g^{-m}\left(z_{0}+n\right)-g^{-m}(w+n)\right)\right|<1 / 5 .
$$

This is a contradiction since $g^{-m}\left(z_{0}+n\right) \in J(f)$ and $\Delta\left(g^{-m}(w+n), 2\right)-k \subset F(f)$. Hence the horizontal line through $z_{0}$ is contained in $J(f)$. Thus $J(f)$ consists of a collection of horizontal lines and $F(f)$ consists of horizontal strips, possibly unbounded. We see that $J(f)=\mathbb{R} \cup\{\infty\}$ as follows. By [3], Theorem 5.6.2, $F(f)$ contains $0,1,2$ or infinitely many components. Our assumptions rule out 0 or 1 components. If there are infinitely many components, we see that $\infty$ is on the boundary of infinitely many components of $F(f)$. As $f^{-1}(\infty)$ must contain a finite preimage $w$ and $F(f)$ is completely invariant under $f$, the point $w$ must also be on the boundary of infinitely many components of $F(f)$. This is obviously impossible from the characterization of $F(f)$ described above. Hence $J(f)$ contains a single horizontal line. Where defined, $g$ maps $J(f)$ into itself and since $g(z)=$ $z / \lambda+O(1 / z)$ with $\lambda>1$, we see that $J(f)=\mathbb{R} \cup\{\infty\}$ as claimed.

We have proven Theorem 1 in the case where infinity or the image of infinity belongs to a repelling cycle. We next treat the case where infinity or the image of infinity belongs to a rationally indifferent cycle. Again, by passing to an iterate 
we may assume that $w_{0}=f(\infty)$ is fixed by $f$ and the multiplier at $w_{0}$ is 1 . See $[3], \S 6.5$, for a treatment of the geometry and dynamics associated with rationally indifferent cycles.

Lemma 5. Let $f$ be a rational function of degree at least two such that $J(f)+$ $1=J(f)$ and $f(\infty)=w_{0}$ is a parabolic fixed point for $f$. If $f(\infty)=\infty$, let $g(z)=f(z)=z+O(1)$ in a neighborhood of infinity and if $f(\infty)=w_{0} \in \mathbb{C}$ let $g$ satisfy $f_{\infty} \circ g=f_{w_{0}} \circ f_{\infty}$ as in (2.7) or (2.10). Where defined, $g$ and $g^{-1}$ leave $J(f)$ and $F(f)$ invariant. Then the function $g$ has exactly two petals at infinity.

Proof. First some comments on the second possible definition of $g$. Since $f_{\infty}(z)=$ $w_{0}+a / z^{k}+O\left(1 / z^{k+1}\right)$ for some $k \geq 1$ and $f_{\infty} \circ g=f_{w_{0}} \circ f_{\infty}$, if $f$ has $p$ petals at $w_{0}$, i.e., $f_{w_{0}}(z)=z+b\left(z-w_{0}\right)^{p+1}+O\left(\left(z-w_{0}\right)^{p+2}\right)$, then $g$ has $k p$ petals at infinity.

The iterates $\left\{g^{n}\right\}$ form a normal family on petals $P_{j}$ at infinity. Since $f_{\infty} \circ g^{n}=$ $f_{w_{0}}^{n} \circ f_{\infty}$ where defined, the iterates $\left\{f_{w_{0}}^{n}\right\}$ form a normal family on $f_{\infty}\left(P_{j}\right)$. Hence $P_{j} \subset F(f)$ by the invariance of the Fatou set under $f$.

At a parabolic fixed point for an analytic function with $q$ petals, each petal subtends an angle of $2 \pi / q$ at the fixed point. If $g$ had one petal at infinity, the invariance of $F(f)$ under $z \mapsto z+1$ would imply that $\mathbb{C} \subset F(f)$, which is an impossibility. If there were $q \geq 2$ petals, any petal under translation will eventually either fill out all of $\mathbb{C}$ as above, or fill out a half-plane, and hence each petal for $g$ is contained in one of two components, $V_{1}$ and $V_{2}$, of $F(f)$. We claim that $q=k p=2$. If $k=1$, then either $g=f$ or $g$ is a local conformal conjugate of $f$ in a neighborhood of the parabolic fixed point $w_{0}$. Then each $P_{j}$ corresponds to a petal for $f$ at $w_{0}$. In particular, $f\left(P_{j}\right)=\Pi_{j}$ for a petal $\Pi_{j}$ for $f$ at $w_{0}$. But $f\left(P_{j}\right)$ is contained in either component $f\left(V_{1}\right)$ or $f\left(V_{2}\right)$ of $F(f)$, and each $\Pi_{j}$ is contained in a distinct component of $F(f)$. Hence $p=2$ and $g$ has exactly two petals at infinity. Now assume $k \geq 2$. From the geometry of the Fatou set at a parabolic fixed point with $p$ petals, if $p \geq 2$ or $k \geq 3$, then the preimage of $J(f)$ under $f_{\infty}^{-1}$ in a neighborhood of $w_{0}$ must intersect any half-plane of the form $\{z: \operatorname{Im}(z)>c\}$ or $\{z: \operatorname{Im}(z)<-c\}$ for all sufficiently large positive $c$ which is impossible since such half-planes are contained in $F(f)$. Thus $p=1$ and $k=2$. Again, $g$ has exactly two petals at infinity.

Remark 5. An analytic function $h$ with parabolic fixed point at 0 with two petals has the expansion $h(z)=z+a z^{3}+O\left(z^{4}\right), a \neq 0$, in a neighborhood of 0 . Conjugating this by $z \mapsto 1 / z$ we see that $g$ has the expansion

$$
g(z)=z-a / z+v(z)
$$

where $|v(z)| \leq C_{0} /\left|z^{2}\right|$ when $|z|>M$ for suitable positive numbers $C_{0}$ and $M$.

We can gain more information about the estimate for $g$ in a neighborhood of infinity.

Lemma 6. Let $f$ and $g$ be as in Lemma 5 such that $g$ has the estimate

$$
g(z)=z-a / z+v(z)
$$

for $|z|>M$ where $|v(z)| \leq C_{0} /\left|z^{2}\right|$ for suitable positive numbers $C_{0}$ and $M$. Then $a>0$. 
Proof. Assume, for the moment, that $a>0$. Let $k=\max \left\{2 C_{0} / a, M\right\}$. Let

$$
\begin{gathered}
H_{1}=\{x+i y: y>k\}, \\
H_{2}=\{x+i y: y<-k\} .
\end{gathered}
$$

A simple calculation gives that

$$
\operatorname{Im}(g(x+i y))=y+\frac{a y}{x^{2}+y^{2}}+\operatorname{Im}(v(x+i y))
$$

and for $z=x+i y \in H_{1}$ we see that

$$
|\operatorname{Im}(v(x+i y))| \leq|v(x+i y)| \leq \frac{C_{0}}{x^{2}+y^{2}}<\frac{a y}{2\left(x^{2}+y^{2}\right)} .
$$

In particular, on $H_{1}$ we have that $\operatorname{Im}(g(x+i y))>y+a y / 2\left(x^{2}+y^{2}\right)>k$. Hence $g\left(H_{1}\right) \subset H_{1}$. A similar estimate on $H_{2}$ gives $g\left(H_{2}\right) \subset H_{2}$. By Montel's Theorem, the family $\left\{g^{n}\right\}$ is normal on $H=H_{1} \cup H_{2}$. Hence as noted in the proof of Lemma 5 , we see that $H \subset F(f)$.

Now consider the general case for $g(z)=z-a / z+v(z)$, where $a=r e^{i \theta}$ with $r>0$ and we may assume $0<\theta<2 \pi$. Setting $\phi(z)=e^{-i \theta / 2} z$ we conjugate $g$ to form the analytic function $h(z)=\phi \circ g \circ \phi^{-1}(z)=z-r / z+\phi \circ v \circ \phi^{-1}(z)$. By the above, the family $\left\{h^{n}\right\}$ is normal on $H$ for a suitably constructed set $H$. Then the family $\left\{g^{n}\right\}$ is normal on $\phi^{-1}(H)$. Hence $\phi^{-1}(H) \subset F(f)$. However, the assumption that $F(f)+1=F(f)$ would then imply that $\mathbb{C} \subset F(f)$, contradicting the fact that $J(f)$ is infinite for all rational functions $f$ with $\operatorname{deg} f \geq 2$. Hence $a>0$ as claimed.

We are now ready to finish off the case when infinity belongs to, or iterates into, a rationally indifferent cycle.

Lemma 7. Let $f$ be a rational function of degree at least two such that $J(f)+1=$ $J(f)$ and $w_{0}=f(\infty)$ is a parabolic fixed point. Then $J(f)=\left\{t+i y_{0}: t \in \mathbb{R}\right\} \cup\{\infty\}$ for some $y_{0} \in \mathbb{R}$.

Proof. Recall that, by Lemma 1, the Julia set of a rational function which is a horizontal line cannot contain a critical point. Hence the assumptions that $J(f)+1=J(f)$ and infinity or the image of infinity is a parabolic fixed point are incompatible with the assumption that infinity is a critical point.

Recall that $g$, defined as in Lemma 5, has the estimate

$$
g(z)=z-\frac{a}{z}+\frac{b}{z^{2}}+w(z),
$$

with $a>0$ where $|w(z)| \leq C_{1} /|z|^{3}$ for $|z|>M_{1}$.

We first show that $J(f)$ is contained in a horizontal line. Assume that this is not the case. The invariance of $J(f)$ under translation by one and the fact that each component of $F(f)$ must contain half-planes described in the proof of Lemma 6 show that there must exist points $z_{1}$ and $z_{2}$ in $J(f)$ on which $\operatorname{Im}(z)$ achieves a maximum and minimum, respectively. Further, we may write $z_{1}=x_{1}+i y_{1}, z_{2}=x_{1}+t+i y_{2}$ where $|t|<1$ and $y_{1}>y_{2}$. Since $J(f)=J(f)+1$, we may take $x_{1}$ to be positive and so large that $g\left(z_{1}\right), g\left(z_{2}\right)$ are defined and can be estimated. We wish to show that

$$
\operatorname{Im}\left(g\left(z_{1}\right)-g\left(z_{2}\right)\right)>y_{1}-y_{2}
$$


for suitably chosen $z_{i}$, which contradicts the extremality of the choice of the $z_{i}$ since $g$ preserves $J(f)$ where defined.

Replace $z_{i}$ by $z_{i}+n$ where the positive integer $n$ is chosen large enough so that $\operatorname{Re}\left(z_{i}\right)>M_{1}$. We evaluate

$$
g\left(z_{1}\right)-g\left(z_{2}\right)=z_{1}-z_{2}-a\left(\frac{1}{z_{1}}-\frac{1}{z_{2}}\right)+b\left(\frac{1}{z_{1}^{2}}-\frac{1}{z_{2}^{2}}\right)+w\left(z_{1}\right)-w\left(z_{2}\right) .
$$

Then

$$
\begin{aligned}
\operatorname{Im}\left(\frac{a}{z_{2}}-\frac{a}{z_{1}}\right) & =a\left(\frac{y_{1}}{x_{1}^{2}+y_{1}^{2}}-\frac{y_{2}}{\left(x_{1}+t\right)^{2}+y_{2}^{2}}\right) \\
& =\frac{a}{x_{1}^{2}}\left(\frac{y_{1}}{1+\left(\frac{y_{1}}{x_{1}}\right)^{2}}-\frac{y_{2}}{\left(1+\frac{t}{x_{1}}\right)^{2}+\left(\frac{y_{2}}{x_{1}}\right)^{2}}\right) .
\end{aligned}
$$

As $a>0$ and $y_{1}-y_{2}>0$, we may choose $x_{1}$ large enough so that the above quantity is positive and of the order of magnitude $1 / x_{1}^{2}$. Further,

$$
\begin{aligned}
b\left(\frac{1}{z_{1}^{2}}-\frac{1}{z_{2}^{2}}\right) & =b \frac{z_{1}+z_{2}}{z_{1}^{2} z_{2}^{2}}\left(z_{2}-z_{1}\right) \\
& =b \frac{2 x_{1}+t+i\left(y_{1}+y_{2}\right)}{x_{1}^{4}\left(1+i \frac{y_{1}}{x_{1}}\right)^{2}\left(1+\frac{t}{x_{1}}+i \frac{y_{2}}{x_{1}}\right)^{2}}\left(t+i\left(y_{2}-y_{1}\right)\right) .
\end{aligned}
$$

Hence

$$
b\left(\frac{1}{z_{1}^{2}}-\frac{1}{z_{2}^{2}}\right)+w\left(z_{1}\right)-w\left(z_{2}\right)=O\left(\frac{1}{x_{1}^{3}}\right)
$$

as $x_{1} \rightarrow+\infty$ and so we may choose $x_{1}$ large enough so that $\operatorname{Im}\left(g\left(z_{1}\right)-g\left(z_{2}\right)\right)>$ $y_{1}-y_{2}$, giving the desired contradiction.

Hence $J(f) \backslash\{\infty\}$ is contained in a horizontal line. If $F(f)$ has exactly two components, then $J(f)$ is a horizontal line.

Now we show that $F(f)$ cannot be connected. Assume that $J(f)$ is a disconnected subset of a horizontal line, i.e., $F(f)$ is connected. By the invariance of $J(f)$ under translation by 1 , there exist a point $z_{0}=x_{0}+i y_{0} \in J(f) \backslash\{\infty\}$ and a maximal positive number $t_{0}$ with $0<t_{0} \leq 1$ such that $z_{0}+t \in F(f)$ for all $0<t<t_{0}$ and $z_{0}+t_{0} \in J(f)$. We may assume that $\operatorname{Re}\left(z_{0}\right)$ is large enough so that $g$ is well defined for $\left\{z:|z|>\operatorname{Re}\left(z_{0}\right) / 2\right\}$. We will show that $z_{0}$ may be chosen so that $\operatorname{Re}\left(g\left(z_{0}+t_{0}\right)-g\left(z_{0}\right)\right)>t_{0}$. However since $g$ is close to the identity near infinity, we would then have that $g\left(z_{0}\right)+t \in F(f)$ for all $t$ with $0<t<t_{1}$, for some $t_{1}>t_{0}$, contradicting the maximality of $t_{0}$. The calculations proceed as above.

Thus

$$
\begin{aligned}
g\left(z_{0}\right. & \left.+t_{0}\right)-g\left(z_{0}\right) \\
& =t_{0}-a\left(\frac{1}{z_{0}+t_{0}}-\frac{1}{z_{0}}\right)+b\left(\frac{1}{\left(z_{0}+t_{0}\right)^{2}}-\frac{1}{z_{0}^{2}}\right)+w\left(z_{0}+t_{0}\right)-w\left(z_{0}\right) .
\end{aligned}
$$


Since

$$
\begin{aligned}
\operatorname{Re}\left(\frac{a}{z_{0}}-\frac{a}{z_{0}+t_{0}}\right) & =a\left(\frac{x_{0}}{x_{0}^{2}+y_{0}^{2}}-\frac{x_{0}+t_{0}}{\left(x_{0}+t_{0}\right)^{2}+y_{0}^{2}}\right) \\
& =a\left(\frac{x_{0}^{2} t_{0}+x_{0} t_{0}^{2}-t_{0} y_{0}^{2}}{\left(x_{0}^{2}+y_{0}^{2}\right)\left(\left(x_{0}+t_{0}\right)^{2}+y_{0}^{2}\right)}\right) \\
& =\frac{a}{x_{0}^{2}}\left(\frac{t_{0}+t_{0}^{2} / x_{0}-t_{0} y_{0}^{2} / x_{0}^{2}}{\left(1+\left(y_{0} / x_{0}\right)^{2}\right)\left(\left(1+t_{0} / x_{0}\right)^{2}+\left(y_{0} / x_{0}\right)^{2}\right)}\right)
\end{aligned}
$$

and $a>0$ and $t_{0}>0$, we may choose $x_{0}$ large enough by replacing $z_{0}$ with $z_{0}+n$ so that the above quantity is positive and is $O\left(1 / x_{0}^{2}\right)$. Again,

Hence

$$
\begin{aligned}
b\left(\frac{1}{\left(z_{0}+t_{0}\right)^{2}}-\frac{1}{z_{0}^{2}}\right) & =-t_{0} b \frac{2 z_{0}+t_{0}}{\left(z_{0}+t_{0}\right)^{2} z_{0}^{2}} \\
& =-t_{0} b \frac{2 x_{0}+t_{0}+2 i y_{0}}{x_{0}^{4}\left(1+\frac{t_{0}}{x_{0}}+i \frac{y_{0}}{x_{0}}\right)^{2}\left(1+i \frac{y_{0}}{x_{0}}\right)^{2}} .
\end{aligned}
$$

$$
b\left(\frac{1}{\left(z_{0}+t_{0}\right)^{2}}-\frac{1}{z_{0}^{2}}\right)+w\left(z_{0}+t_{0}\right)-w\left(z_{0}\right)=O\left(\frac{1}{x_{0}^{3}}\right)
$$

as $x_{0} \rightarrow \infty$. Hence we may choose $x_{0}$ large enough so that

$$
\operatorname{Re}\left(g\left(z_{0}+t_{0}\right)-g\left(z_{0}\right)\right)>t_{0}
$$

giving the desired contradiction.

Hence if $J(f)+1=J(f)$ and infinity or the image of infinity is a parabolic fixed point, then $f$ is conformal at infinity and $J(f) \backslash\{\infty\}$ is a horizontal line as claimed.

The final case in the proof of Theorem 1 occurs when $w_{0}=f(\infty)$ is a Cremer point, i.e., $w_{0} \in J(f)$ and $f$ has an irrational rotation about $w_{0}$ as its first order approximation.

Lemma 8. Let $f$ be a rational function of degree at least two such that $J(f)+1=$ $J(f)$ and let $g$ be an analytic function such that

$$
g(z)=e^{2 \pi i \theta} z+O(1 / z)=\eta z+O(1 / z)
$$

in a neighborhood of infinity where $0<2 \pi \theta<\pi, \theta$ irrational, and such that where defined, $g$ leaves $J(f)$ invariant. Then $J(f)=\overline{\mathbb{C}}$.

We will use this lemma in the case where $f(\infty)=\infty$ by setting $g=f$ and defining $g$ by (2.7) or (2.10) when $f(\infty)=w_{0} \in \mathbb{C}$ is a Cremer point for $f$.

Proof. Assume that $F(f) \neq \emptyset$, i.e., there is a disk $\Delta=\Delta\left(z_{0}, r\right) \subset F(f)$. From $\Delta$, we form a "grid" of disks contained in $F(f)$. Specifically, replace $\Delta$ with $\Delta+n$ and $z_{0}$ with $z_{0}+n$ where the positive integer $n$ is chosen large enough so that $g(\Delta)$ contains the disk $\Delta\left(\eta z_{0}, r-\epsilon\right)$ and so $F(f)$ contains the disks $\Delta\left(\eta z_{0}+j, r-\epsilon\right)$ for all integers $j$ and where $\epsilon>0$ may be taken as small as we choose. We may also assume that $\operatorname{Im}\left(\eta z_{0}\right)$ is large. Again, $\Delta$ may have been initially chosen so that a further application of $g$ shows that $F(f)$ contains a collection of disks of the form

$$
\{\Delta(w+\eta j+k, r-\epsilon): j, k \in \mathbb{Z}\}
$$

for some $w \in \mathbb{C}$. 
As $\theta$ is irrational, it is well known that the set of multiples $\{n \theta: n=1,2,3, \ldots\}$ is dense modulo 1 in $[0,1]$. Thus we may apply the above construction with some iterate $g^{n}$ where $\left|\eta^{n}-1\right|<(r-\epsilon) / 4$. We show that $F(f)$ contains the strip

$$
S=\left\{z: \operatorname{dist}(z, \mathcal{L})<\frac{r-\epsilon}{4}\right\}
$$

where $\mathcal{L}=\left\{w+t\left(\eta^{n}-1\right): t \in \mathbb{R}\right\}$. Choose any $t \in \mathbb{R}$ and pick an integer $m$ with $|t-m| \leq 1 / 2$. Consider the points $w_{0}=w+t\left(\eta^{n}-1\right)$ and $z_{0}=w+m\left(\eta^{n}-1\right)$. Note that $\left|z_{0}-w_{0}\right|=\left|(m-t)\left(\eta^{n}-1\right)\right|<(r-\epsilon) / 8$. Then if $z$ is any point such that $\left|z-w_{0}\right|<(r-\epsilon) / 4$, then $\left|z-z_{0}\right|<3(r-\epsilon) / 8<r-\epsilon$. In particular,

$$
\Delta\left(w+t\left(\eta^{n}-1\right), \frac{r-\epsilon}{4}\right) \subset \Delta\left(w+m\left(\eta^{n}-1\right), r-\epsilon\right) \subset F(f) .
$$

Thus $S \subset F(f)$ since $t$ was arbitrary.

We now show that, for a suitable positive integer $m, g^{m}(S)$ contains a strip that fills out the plane under integer translations. For a given $m$ we may choose an integer $k$ so that $f^{m}(S+k)$ contains a strip $T$ of the form

$$
T=\left\{z: \operatorname{dist}\left(z, \mathcal{L}^{\prime}\right)<(r-\epsilon) / 8\right\}
$$

where $\mathcal{L}^{\prime}=\left\{w^{\prime}+t \eta^{m}\left(\eta^{n}-1\right): t \in \mathbb{R}\right\}$. We then choose $m$ so that $\arg \left(\eta^{m}\left(\eta^{n}-1\right)\right)$, which necessarily is non-zero, is so small that $T \cap(T+1) \neq \emptyset$. Thus $\mathbb{C}=\bigcup(T+n) \subset$ $F(f)$. This is impossible as $J(f)$ must contain infinitely many points. Hence $J(f)=\overline{\mathbb{C}}$.

Theorem 1 now follows from Lemmas 4, 7, and 8 .

\section{REFERENCES}

[1] I.N. Baker and A. Eremenko. A problem on Julia sets. Ann. Acad. Sci. Fenn., 12:229-236, 1987. MR 89g:30047

[2] Alan F. Beardon. Symmetries of Julia sets. Bull. London Math. Soc., 22:576-582, 1990. MR 92f:30033

[3] Alan F. Beardon. Iterations of Rational Functions. Springer-Verlag, New York, 1991. MR 92j:30026

[4] G. Levin and F. Przytycki. When do two rational functions have the same Julia set? Proc. Amer. Math. Soc., 125(7):2179-2190, 1997. MR 97i:58149

[5] G.M. Levin. Symmetries on the Julia set. Math. Notes, 48.5-6:1126-1131, 1991. MR 92e:30015

Department of Mathematics, University of Illinois, Urbana, Illinois 61801

E-mail address: boyd@math.uiuc.edu

Current address: Department of Mathematics, Purdue University, West Lafayette, Indiana 47907

E-mail address: boyd@math.purdue.edu 